\title{
A note on planes of characteristic three
}

\author{
Michael J. Kallaher
}

\begin{abstract}
A projective plane has characteristic three if in every ternary ring coordinatizing it all elements $\neq 0$ of the additive loop have order 3 . We show that if a finite plane of characteristic three is coordinatized by a near-field, then the plane is desarguesian.
\end{abstract}

\section{Introduction}

Lombardo-Radice [7] has defined a projective plane $\pi$ to have characteristic $m, m$ a positive integer, if in every ternary ring coordinatizing $\pi$ all elements of the additive loop except the identity $c$ have the same order $m$. The only known finite planes with characteristic are the desarguesian ones. It has been conjectured that there are no others. This has been proved only of the case $m=2$ (Gleason [3]).

The case $m=3$ has been investigated by Keedwell $[5,6]$, Lombardo-Radice [7,8], and Zappa [9]. Lombardo-Radice shows that a plane has characteristic 3 if and only if every quadrangle generates a subplane isomorphic to the plane of order 3 . Zappa investigates the question of existence of characteristic 3 planes. He proves that if the plane is coordinatized by a near-field of dimension 2 or less over its center. then the plane is desarguesian. He also shows that no Hughes plane has characteristic 3 .

Keedwell derives several configuration theorems associated with the idea of characteristic and does a detailed investigation of them.

Received 8 ilarch 1972. The author acknowledges part support from the National Science Foundation of the USA. 
In this paper we extend Zappa's first result to all finite near-fields; that is, we prove:

MAIN THEOREM. If a finite plane $\pi$ of characteristic 3 is coordinatized by a near-field, then $\pi$ is desarguesian.

The idea of the proof is as follows. Every near-field, except the seven exceptional ones, is a generalized Andre system. Using this fact we are able to prove (Theorem A) that if a near-field satisfies

$$
(1+x) x=x+x^{2},
$$

then it is a field. We then show (Theorem $B$ ) that this identity is satisfied in a near-field coordinatizing a plane of characteristic 3 .

We assume the reader is completely familiar with the theory of projective planes as given in Chapters 3-5 of Dembowski [1].

\section{Proof of the main theorem}

A generalized André system [2] is a quasi-field $(Q,+\cdot)$ defined as follows: $(Q,+)=\left(G F\left(p^{r}\right),+\right)$ and $" \cdot "$ is defined by

$$
\begin{aligned}
& m \cdot x=m x^{p^{\lambda(m)}}, m \neq 0, \\
& 0 \cdot x=0,
\end{aligned}
$$

where $\lambda$ is a mapping from the multiplicative group $G F\left(p^{r}\right)-\{0\}$ into the additive group $z_{r}$ and multiplication on the right in ( 1 ) is multiplication in $G F\left(p^{r}\right) . \lambda$ also satisfies the two restrictions:

(i) $\lambda(1)=0$;

(ii) if $x=\omega^{i}, y=\omega^{j}$ with $i \equiv j\left(\bmod p^{t}-1\right)$ where $t=$ g.c.d. $(r, \lambda(x)-\lambda(y))$ then $x=y$. (Here $\omega$ is a fixed primitive element of $G F\left(p^{r}\right)$.)

The non-exceptional finite near-fields are all generalized André systems ([2], p. '383). Note that a generalized André system is a near-field if and only if the mapping $\lambda$ satisfies

$$
\lambda(a \cdot b)=\lambda(a)+\lambda(b)
$$


for all $a, b \in \mathrm{GF}\left(p^{2}\right)-\{0\}$. Also if $\lambda(a)=0$ for all $a \neq 0$ then $Q$ is the field GF $\left(p^{2}\right)$.

THEOREM A. If $(Q,+, \cdot)$ is a finite near-field in which

$$
(1+x) x=x+x^{2}
$$

for alz $x \in Q$, then $(Q,+, \cdot)$ is a field.

Proof. Assume first that $(Q,+, \cdot)$ is one of the exceptional finite near-fields. If $u$ is the element satisfyịg $u^{2}=-1$ (and there is such an element in each of the seven exceptional near-fields - Hall [4]), then $(1+u) u \neq u+u^{2}$. This follows from an easy calculation using the identities in $\mathrm{Hall}$ [4].

Assume now that $(Q,+, \cdot)$ is a non-exceptional near-field. Then $(Q,+, \cdot)$ is a generalized André system in which (2) is satisfied. For all $a, x \in Q-\{0\}$ we have

$$
[a \cdot(1+x)] \cdot x=a \cdot[(1+x) \cdot x],
$$

which becomes

$$
\begin{aligned}
& {\left[a+a x^{p^{\lambda(a)}}\right] x^{p^{\lambda(a)+\lambda(1+x)}}=a\left(x+x x^{p^{\lambda(x)}}\right)^{p^{\lambda(a)}}} \\
& a\left(1+x^{p^{\lambda(a)}}\right) x^{p^{\lambda(a)+\lambda(1+x)}}=a\left(1+x p^{\lambda(x)+\lambda(a)}\right) \cdot p^{\lambda(a)},
\end{aligned}
$$

where multiplication is now in $G F\left(p^{r}\right)$. Hence we have

$$
\left[1+x^{p^{\lambda(a)}}\right] x^{p^{\lambda(1+x)}}=1+x^{p^{\lambda(x)+\lambda(a)}}
$$

for all $a, x \in \mathrm{GF}\left(p^{r}\right)-\{0\}$.

Assume there exists an $a \in G F\left(p^{r}\right)-\{0\}$ with $\lambda(a) \neq 0$. If $\lambda(x)=0$ then

$$
x^{p^{\lambda(1+x)}}=1
$$

which implies $x=1$. Since (2) implies $\lambda$ is a (group) homomorphism from the multiplicative group of $Q$ into the additive group $z_{r}$ this last equation implies $\lambda$ is an isomorphism. Since $|Q-\{0\}|=p^{r}-1$ and 
$\left|z_{r}\right|=r$ we must have $p^{r}-1 \leq r$ and this occurs only if $p=2$, $r=1$. However if $p^{x}=2, \lambda(a)=0$ always.

Thus we have shown that $\lambda(a)=0$ for all $a \in G F\left(p^{p}\right)-\{0\}$ and this implies $Q$ is $\mathrm{GF}\left(p^{r}\right)$.

THEOREM B (Zappa [9]). Let $\pi$ be a finite plane of characteristic 3 which is coordinatized by a near-field $(Q,+, \cdot)$ with respect to the points $U, V, O, I$. For all $u \in Q$ we have

$$
(1+u) u=u+u^{2} \text {. }
$$

Proof. We remark that for all $y \in Q, y+y+y=0$ or $y+y=-y$. We assume now that $Q$ has dimension $r>1$ over $\mathrm{GF}(3)$; that is, $\pi$ has order $3^{r}, r>1$. We need only prove the theorem for $u \neq 0,1,-1$. Consider the quadrangle with vertices $0=(0,0)$, $P_{1}=(1,0), P_{2}=(0,1), R=(u, u)$. The points on the lines $O P_{1}$, $O P_{2}, O R, P_{1} P_{2}, P_{1} R, P_{2} R$ satisfy the following equations

$$
\begin{aligned}
& O P_{1}: y=0, O P_{2}: x=0, O R: y=x, \\
& P_{1} P_{2}: y=-x+1, \\
& P_{1} R: y=u(u-1)^{-1} x-u(u-1)^{-1}, \\
& P_{2} R: y=(u-1) u^{-1} x+1,
\end{aligned}
$$




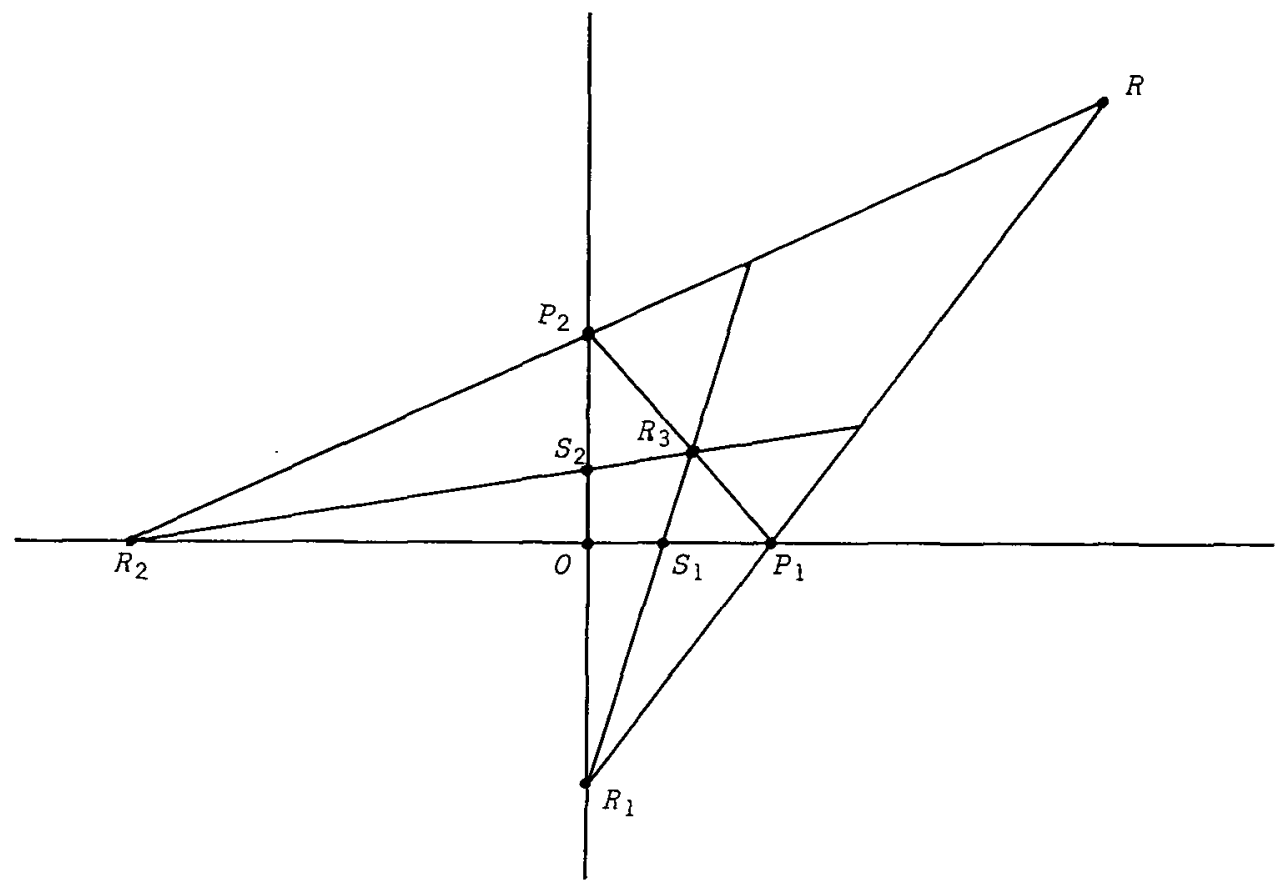

Let $R_{1}=P_{1} R \cap O P_{2}, R_{2}=O P_{1} \cap P_{2} R, R_{3}=O R \cap P_{1} P_{2}$. Then we have

$$
\begin{gathered}
R_{1}=\left(0,-u(u-1)^{-1}\right), R_{2}=\left(-u(u-1)^{-1}, 0\right), \\
R_{3}=(-1,-1) .
\end{gathered}
$$

The lines $R_{1} R_{3}$ and $R_{2} R_{3}$ have the equations

$$
\begin{gathered}
R_{1} R_{3}: y=\left[1-u(u-1)^{-1}\right] x-u(u-1)^{-1}, \\
R_{2} R_{3}: y=\left[1-u(u-1)^{-1}\right] x+\left[(u-1) u^{-1}-1\right]^{-1} . \\
\text { If } S_{1}=O P_{1} \cap R_{1} R_{3} \text { and } S_{2}=O P_{2} \cap R_{2} R_{3}, \text { then } \\
S_{1}=\left(\left[(u-1) u^{-1}-1\right]^{-1}, 0\right), S_{2}=\left(0,\left[(u-1) u^{-1}-1\right]^{-1}\right) .
\end{gathered}
$$

Thus $S_{I} S_{2}$ has the equation 


$$
S_{1} S_{2}: y=-x+\left[(u-1)^{-1}-1\right]^{-1} \text {. }
$$

Since $O, P_{1}, P_{2}, R$ generate a subplane isomorphic to the plane of order 3 , we know the point $R$ lies on the line $S_{I} S_{2} \cdot$ This gives

$$
u=-u+\left[(u-1) u^{-1}-1\right]^{-1} \text {, }
$$

which becomes

$$
\begin{aligned}
u & =\left[1-(u-1) u^{-1}\right]^{-1}, \\
u^{-1} & =1-(u-1) u^{-1}, \\
(u-1) u^{-1} & =1-u^{-1} .
\end{aligned}
$$

Because $(Q,+, \cdot)$ is. a near-field we have

$$
u^{-1}(u-1)=1-u^{-1} \text {. }
$$

Thus

$$
(u-1) u^{-1}=u^{-1}(u-1)
$$

which gives

$$
u(u-1)=(u-1) u
$$

If $v=u-1$, then we have

$$
(v+1) v=v(v+1)=v^{2}+v
$$

for all $v \in Q$ with $v \neq 0,1,-1$. This proves the theorem.

We can now prove the main theorem. By Theorem $B$ the near-field $(Q,+, \cdot)$ satisfies the identity $(1+u) u=u+u^{2}$ for all $u \in Q$. By Theorem $A,(Q,+, \cdot)$ is a field. Hence $\pi$ is desarguesian.

\section{References}

[1] P. Dembowski, Finite geometries (Ergebnisse der Mathematik und ihrer Grenzgebiete, Band 44. Springer-Verlag, Berlin, Heidelberg, New York, 1968). 
[2] David A. Foulser, "A generalization of André's systems", Math. 2. 100 $(1967), 380-395$.

[3] Andrew M. Gleason, "Finite Fano planes", Amer. J. Math. 78 (1956), 797-807.

[4] Marshall Hall, Jr, Combinatorial theory (Blaisdell Publishing Co. [Ginn and Co.], Waltham, Massachusetts; Toronto, Ontario; London; 1967).

[5] A.D. Keedwell, "On the order of projective planes with characteristic", Rend. Mat. e Appl. (5) 22 (1963), 498-530.

[6] A.D. Keedwell, "A class of configurations associated with projective planes with characteristic", Arch. Math. 15 (1964), 470-480.

[7] Lucio Lombardo-Radice, "Sul rango dei piani grafici finite a caratteristice 3 ", Bolz. Un. Mat. Ital. (3) 10 (1955), 172-177.

[8] Lucio Lombardo-Radice, "Su alcuni caratteri dei piani grafici", Rend. Sem. Mat. Univ. Padova 24 (1955), 312-345.

[9] Guido Zappa, "Piani grafici a caratteristica 3 ", Ann. Mat. Pura App Z. (4) 49 (1960), 157-166.

Department of Pure and Applied Mathematics, Washington State University,

Pul Iman, Washington, USA. 